\title{
Recomendações para o tratamento da filariose bancroftiana, na infecção e na doença
}

\author{
Recommendations for the treatment of bancroftian filariasis \\ in symptomless and diseased patients
}

Gerusa Dreyer ${ }^{2}$, Patrícia Dreyer ${ }^{1}$ e Joaquim Norões ${ }^{13}$

\begin{abstract}
Resumo As bases para se instituir o tratamento da filariose linfática são: prevenir, reverter ou diminuir a progressão da doença e interromper a transmissão do parasita. A escolha das medidas mais adequadas para o tratamento dos pacientes portadores de filariose linfática requer o conhecimento das diversas manifestações clínicas, assim como os seus diferentes mecanismos patogênicos. No passado, o tratamento da filariose era baseado somente na prescrição da droga antifilarial, porém, para os pacientes apresentando as manifestações agudas ou crônicas da doença, é imperativo que outras medidas sejam indicadas, incluindo higiene e dieta. Assim, essa nova visão multidisciplinar terapêutica é tão ou mais importante do que o início imediato da terapia antifilarial. Independentemente da manifestação clínica do paciente, três componentes estão agora indicados: 1) os cuidados clínicos e higiênico-dietéticos específicos para as diversas manifestações clínicas, 2) a educação e o suporte psicológico e finalmente 3) o tratamento quimioterápico com a dietilcarbamazina (DEC) ou a combinação de DEC e ivermectina. Os autores descrevem também as eficácias relativas macro e microfilaricidas da dietilcarbamazina e da ivermectina sozinhas ou em combinação usadas em programas de tratamento em massa para interrupção da transmissão e comentam sobre a introdução do uso da higiene em programas de saúde pública para a prevenção do linfedema.
\end{abstract}

Palavras-chaves: Controle. Tratamento. Dietilcarbamazina. Ivermectina. Bancroftose. Elefantíase.

Abstract The goals of treatment for lymphatic filariasis are: to prevent, reverse, or halt progression of disease; and to interrupt transmission of the parasite. Selecting the appropriate therapy for the patient with lymphatic filariasis requires knowledge of the various clinical features of filarial disease and their pathogenesis. In the past, treatment of lymphatic filariasis has focused primarily on antiparasitic chemotherapy; however, for many of the acute and chronic manifestations of lymphatic filariasis, it is now clear that supportive or other forms of clinical care are even more important than antiparasitic medication in order to prevent worsening of the disease. Regardless of the clinical manifestations of filarial disease in a particular patient, the following three components of treatment should, in general, be considered: supportive or disease-specific clinical care (including higiene and diet), patient education and counseling and finally, antiparasitic chemotherapy with diethylcarbamazine (DEC) and/or the combination of DEC with ivermectin. The authors also describe the proportional efficacy of diethylcarbamazine and ivermectin, alone or in combination, for use in mass treatment aiming at transmission interruption and the use of hygiene as a public health approach for lymphedema prevention.

Key-words: Control. Treatment. Diethylcarbamazine. Ivermectin. Bancroftian filariasis. Elephantiasis.

Nos últimos anos, a filariose linfática tem surgido como um problema crescente em saúde pública em várias partes do mundo ${ }^{36}$. A debilitação física e o estigma social associados à filariose linfática têm sido considerados como a segunda causa mundial de incapacidade para o trabalho ${ }^{63}$.
Nas áreas em que a doença é endêmica, a prevalência de infecção aumenta continuadamente. Isso ocorre, principalmente, pelo crescimento rápido e desordenado dos centros urbanos e pela criação de condições ideais para a multiplicação dos focos de mosquitos transmissores da doença, sendo o

1. Núcleo de Ensino, Pesquisa e Assistência em Filariose (NEPAF) do Hospital das Clínicas da Universidade Federal de Pernambuco (UFPE), Recife; 2. Centro de Pesquisas Aggeu Magalhães da Fundação Oswaldo Cruz (FIOCRUZ), Recife, 3. Departamento de Cirurgia do Centro de Ciências da Saúde da Universidade Federal de Pernambuco (UFPE), Recife, PE, Brasil.

Endereço para correspondência: Prof ${ }^{a}$ Gerusa Dreyer. NEPAF/Hospital das Clínicas/UFPE. Av. Prof. Moraes Rego s/n, 5o andar, Cidade Universitária, 50740-900 Recife, PE, Brasil.

Tel: 5581 3454-36 49; Fax: $55813242-7307$

Recebido para publicação em 28/5/2001. 
Culex quinquefasciatus predominante na maioria das regiões endêmicas. Assim, a bancroftose é considerada hoje uma doença reemergente ${ }^{20}$. $O$ desenvolvimento de metodologias de diagnóstico individual e de tratamento em massa tem proporcionado novos horizontes no sentido de eliminar globalmente a W. bancrofti. Em 1993, a Força Tarefa para Erradicação de Doenças elegeu a filariose linfática como uma das seis doenças potencialmente elimináveis ${ }^{10}$. Em maio de 1997, a Assembléia Mundial da Saúde consolidou essa resolução, anunciando a eliminação global da filariose como um problema de saúde pública ${ }^{62}$. Em julho de 1997, em Townsville, Queensland, Austrália, representantes da OMS, de seus centros colaboradores, ministérios de saúde, organizações não governamentais, academias científicas e indústrias farmacêuticas estabeleceram que o controle da filariose seria feito baseado em dois grandes pilares: a interrupção da transmissão e o controle da morbidade ${ }^{56}$. As bases científicas para a nova metodologia do tratamento nos seus diversos níveis foram revistas recentemente por Dreyer \& Dreyer, 200018.

\section{TRATAMENTO QUIMIOTERÁPICO ANTIFILARIAL}

Tratamento individual da infecção. $O$ tratamento antifilarial está indicado em todos os indivíduos com evidências de infecção ativa (vermes adultos e/ou microfilárias), independentemente de apresentarem ou não qualquer manifestação clínica relacionada direta ou indiretamente à bancroftose. A droga de escolha para o tratamento individual é a dietilcarbamazina (DEC*). Infelizmente, o poder adulticida da droga não é $100 \%$ e estima-se que cerca de 50 a $60 \%$ dos vermes adultos são refratários ao tratamento ${ }^{51}$. Através do uso da ultrassonografia, foi possível definir a existência de ninhos de vermes com subpopulações sensíveis e outras que não respondiam ao tratamento com a DEC. Isso acontece mesmo quando se administram doses tão altas da droga quanto $12 \mathrm{mg} / \mathrm{kg}$ de peso corporal, por um período de até 30 dias $^{51}$. Até o momento, não se tem uma explicação exata para esse mecanismo de falha. Assim, a cura parasitológica de um dado indivíduo está na dependência da sensibilidade dos vermes à droga e, desse modo, não é possível antever a resposta terapêutica em cada indivíduo. O esquema clássico recomendado pela Organização Mundial de Saúde para o tratamento individual dos microfilarêmicos é o de $6 \mathrm{mg} / \mathrm{kg}$ de peso por doze dias. No caso da eosinofilia pulmonar tropical (EPT), ele deve ser prolongado por até $30 \mathrm{dias}^{27}$. É oportuno ressaltar que essa síndrome pode levar o paciente a óbito pelo desenvolvimento de fibrose intersticial pulmonar. Dessa forma, existindo dúvidas quanto ao diagnóstico diferencial em relação a outras síndromes pulmonares eosinofílicas, o teste terapêutico com a DEC se faz imperativo ${ }^{11}$. É importante frisar que corticoesteróides não estão indicados para o tratamento de EPT, por reduzirem o efeito microfilaricida da DEC ${ }^{55}$ e por proporcionarem uma melhora passageira com agravamento posterior do quadro clínico.

O efeito macrofilaricida da DEC é doseindependente, a partir de $6 \mathrm{mg} / \mathrm{kg}$, em uma única tomada ${ }^{51}$. Portanto, se o tratamento individual objetiva a morte do verme adulto, ele poderá ser feito com a dose única acima recomendada. Até o momento, as tentativas de repetição do tratamento a cada 6 ou 12 meses, por até 4 anos, têm falhado quanto ao propósito de eliminar os vermes adultos que anteriormente foram refratários à ação da droga (G Dreyer: comunicação pessoal). Por outro lado, o efeito microfilaricida da DEC a curto prazo é dose dependente ${ }^{3}$. Assim, nos portadores de hematúria filarial ${ }^{28}$, o tratamento de 12 dias deve ser repetido tantas vezes quantas forem necessárias para levar ao desaparecimento das microfilárias circulantes. Ocorrendo falha no tratamento com a DEC (pelo fato de o paciente apresentar vermes adultos não susceptíveis e continuar produzindo microfilárias) e havendo reaparecimento da hematúria, deve-se lançar mão da co-admininistração da DEC com a ivermectina* * $(6 \mathrm{mg} / \mathrm{kg}$ e $400 \mu \mathrm{g} / \mathrm{kg}$ de peso, respectivamente). Essa indicação tem como objetivo a melhor ação microfilaricida da combinação das duas $\operatorname{drogas}^{16} \mathrm{e}$, conseqüentemente, a obtenção de um maior tempo de clearance das microfilárias circulantes. Maiores detalhes sobre a DEC podem ser encontrados no trabalho de revisão feito por Dreyer e Norões ${ }^{22}$, no qual os autores abordam sua farmacocinética, farmacodinâmica e eficácia terapêutica.

Os efeitos colaterais do tratamento antifilarial, isto é, aqueles que não se relacionam com a morte do parasito são dose-dependentes. Eles inexistem com a ivermectina mas são leves e transitórios quando se usa a DEC. Esses efeitos se caracterizam por sonolência, náusea e malestar gástrico e geralmente desaparecem após o terceiro dia da administração da droga, mesmo sem redução da dose $^{30}$, quando o tratamento de 12 dias é recomendado.

As reações adversas sistêmicas são microfiláriadependentes, assemelham-se para as duas drogas e se caracterizam, principalmente, por hematúria transitória, que geralmente é microscópica, e febre, podendo ainda existir cefaléia e mialgia ${ }^{30}$. Tais reações podem aparecer precocemente quanto em cinco horas, e geralmente desaparecem em torno de 48 horas após

\footnotetext{
" A DEC é derivada da piperazina, distribuída no Brasil pela Fundação Nacional de Saúde e fabricada por Farmanguinhos/FIOCRUZ. É apresentada em comprimidos de $50 \mathrm{mg}$ do sal citratado.

**A Ivermerctina já está comercializada no Brasil pela Sintofarma ${ }^{\circledR}$ com o nome de Revectina. É apresentada em comprimidos de $6 \mathrm{mg}$.
} 
o início do tratamento. Não há como predizer se um determinado indivíduo irá ou não apresentar reações adversas sistêmicas. Porém, uma vez existindo, essas serão tanto mais severas quanto maior for o número de microfilárias circulantes. Comumente, já pode ocorrer reação adversa sistêmica com microfilaremia a partir de $250-300 / \mathrm{ml}$. Em alguns poucos casos, pode haver necessidade de se fazer uso de antitérmicos e analgégicos. No entanto, não há indicação para o uso de corticosteróides com a finalidade de minimizar ou de abolir tais reações ${ }^{46}$. As reações adversas localizadas estão associadas à morte dos vermes adultos e apresentam-se clinicamente como linfangite e adenite. Ocorrem após o uso da DEC ${ }^{1621313251}$ e não são observadas com a ivermectina quando administrada isoladamente ${ }^{1524}$. O processo é conhecido como linfangite filarial aguda (LFA), similar ao que ocorre na morte natural (não induzida por droga) dos parasitas adultos ${ }^{21}$. Os pacientes podem ser assintomáticos ou apresentar queixa de dor, geralmente de pouca intensidade, no local ou nas proximidades onde ocorreu a morte dos vermes. O paciente do sexo masculino, algumas vezes, além da dor, pode notar aumento de volume no conteúdo escrotal, por aparecimento de hidrocele aguda $^{17} 5051$, que se reverte na maioria dos casos. Os quadros de linfangite (sempre retrógrada nos membros superiores e inferiores) e de linfadenite são similares, independentemente de ter ocorrido por morte natural ou provocada pelo tratamento antifilarial. Nos casos sintomáticos, não está indicado o uso de antiinflamatórios, sendo recomendada apenas a utilização local de compressas frias e o repouso do paciente.

Vale ressaltar a severidade da reação adversa local após a morte natural do verme apresentada por algumas mulheres grávidas. Além de dor intensa localizada, podem evoluir com quadro clínico sistêmico semelhante ao de uma infecção bacteriana, apresentando febre alta, calafrios, náusea e até vômitos nos casos mais graves. Além do tratamento sintomático permitido para gestantes, está indicado repouso e uso local de compressas frias até o desaparecimento da dor. Apesar de intenso, o processo agudo não deixa seqüelas crônicas.

A DEC não deve ser administrada em mulheres grávidas (apesar de não existirem relatos na literatura de teratogênese em seres humanos) nem durante o primeiro mês de lactação. Normalmente, pode ser administrada a crianças após o segundo ano de vida e, a partir daí, não existe uma idade limite que impeça a sua utilização. É importante lembrar a possibilidade do aparecimento de reações adversas sistêmicas nos microfilarêmicos idosos, com outras doenças associadas, tais como cardiopatias ou pneumopatias. Esses casos devem ser acompanhados através de monitorização cuidadosa nas primeiras quarenta e oito horas. A Tabela 1 mostra o tratamento antifilarial recomendado para as diversas formas clínicas.

O tratamento em massa - interrupção da transmissão. Pesquisas recentes demonstraram que: 1) a dose única de $6 \mathrm{mg} / \mathrm{kg}$ de DEC é tão efetiva para matar o verme adulto de $W$. bancrofti ${ }^{1}$ e provocar supressão da microfilaremia a longo prazo (12 meses), quanto a dose de $6 \mathrm{mg} / \mathrm{kg}$ por 12 dias, classicamente recomendada pela $\mathrm{OMS}^{3}$; 2) a dose única de ivermectina com $400 \mu \mathrm{g} / \mathrm{kg}$ é, inclusive, melhor que a DEC para suprimir a microfilaremia ${ }^{17}{ }^{42}$ a curto prazo (até 6 meses); 3) a supressão a curto e a longo prazo é aumentada se as duas drogas forem co-adminstradas ${ }^{1749}$; 4) a combinação da DEC ou da ivermectina com o albendazol pode, também, aumentar a supressão da microfilaremia a curto ${ }^{1}$ e a longo prazo ${ }^{39} 53$. Com a administração anual de DEC ou ivermectina em programas de tratamento em massa, há uma redução da prevalência de microfilaremia na população humana, assim como do índice de infectividade dos mosquitos vetores ${ }^{5}$. Adicionalmente ao clearence da microfilaremia, o largo espectro anti-helmíntico da ivermectina e do albendazol é um ponto muito atrativo para os programas de tratamento em massa. O uso da DEC no sal convencional de cozinha é uma boa medida adjuvante na interrupção da transmissão. Essa estratégia foi capaz de consolidar a interrupção da transmissão na China $^{35}$. Estão sendo realizados outros estudos para avaliar a eficácia dessa metodologia em outros países, quer isoladamente, quer complementando-se os ciclos anuais de tratamento supracitados.

$\mathrm{Na}$ África, onde pode existir a infecção por Onchocerca ou por Loa loa, com exceção do Egito, o risco potencial de reações adversas severas com o uso da DEC torna impossível sua distribuição nas regiões endêmicas de bancroftose. Nessas áreas, a coadministração de ivermectina e albendazol é o tratamento de escolha. Para as drogas mencionadas e para as suas diversas combinações, o nível de cobertura populacional e a duração do tratamento requerido para interromper a transmissão não foram ainda estabelecidos de forma precisa nas áreas endêmicas com diferentes espécies de vetores e com diferentes prevalências de microfilaremia. Porém, estima-se um período que pode variar de 5 a 10 anos, exceto para o sal fortificado com DEC, para o qual ainda não está estabelecido o tempo máximo de tratamento.

O controle do vetor, quando possível, pode ajudar a interrupção da transmissão, além de proporcionar uma melhor adesão das comunidades aos programas de controle. Na Tabela 2, encontram-se os diversos esquemas terapêuticos sugeridos para o tratamento em massa.

\section{CONTROLE DA MORBIDADE ASSOCIADA À DISFUNÇÃO LINFÁTICA}

Individual. Experiências do Brasil ${ }^{21}$, da Índia ${ }^{57} 58$, assim como dados preliminares do Haiti (D Addiss: comunicação pessoal). indicam claramente que o linfedema de membros ${ }^{21} 26$ é reversível até certo ponto e que pode ser evitado através de medidas que enfatizem a higiene diária e a fisioterapia, principalmente ativa. Essa mesma metodologia é recomendada para o tratamento do linfedema na 
Tabela 1 - Tratamento antifilarial e de outras medidas gerais recomendados para pacientes adultos com infecção/doença bancroftiana.

\begin{tabular}{|c|c|c|c|c|c|}
\hline \multirow[b]{2}{*}{ Manifestação clínica } & \multirow[b]{2}{*}{$\begin{array}{l}\text { Probabilidade da } \\
\text { infecção ativa }\end{array}$} & \multicolumn{4}{|c|}{ Tratamento recomendado } \\
\hline & & DEC & IV & $\begin{array}{l}\text { Educação e } \\
\text { aconselhamento }\end{array}$ & Outros procedimentosgerais/medicamentos \\
\hline \multicolumn{6}{|l|}{ Assintomáticos ou não } \\
\hline microfilarêmicos & $100 \%$ & $\operatorname{sim}^{*}$ & NR & $\operatorname{sim}$ & $\begin{array}{l}\text { ultra-som de bolsa escrotal para determinar } \\
\text { a eficácia do tratamento contra os vermes adultos }\end{array}$ \\
\hline \multicolumn{6}{|c|}{ amicrofilarêmicos portadores } \\
\hline de vermes adultos & $100 \%$ & $\operatorname{sim}^{*}$ & NR & $\operatorname{sim}$ & $\begin{array}{l}\text { ultra-som de bolsa escrotal para determinar a } \\
\text { eficácia do tratamento contra os vermes adultos }\end{array}$ \\
\hline $\begin{array}{l}\text { Microfilarêmicos } \\
\text { com hematúria }\end{array}$ & $100 \%$ & $\begin{array}{c}12 \text { dias } \\
\text { (ver texto) }\end{array}$ & $\begin{array}{l}\operatorname{Sim} \\
\text { (ver texto) }\end{array}$ & $\operatorname{sim}$ & monitorizar a hematúria \\
\hline \multicolumn{6}{|l|}{ Episódios agudos } \\
\hline LFA $^{* *}$ & $100 \%$ & $\begin{array}{l}\operatorname{sim}^{*} \text { após } \\
\text { episódio agudo }\end{array}$ & NR & $\operatorname{sim}$ & compressas frias, repouso \\
\hline $\mathrm{DLAA}^{* * *}$ & muito baixa & $\begin{array}{l}\operatorname{sim}^{*} \text { se } \\
\text { infectado }\end{array}$ & NR & $\operatorname{sim}$ & $\begin{array}{l}\text { compressas frias, analgésicos, antipiréticos, } \\
\text { antibióticos e antifúngicos tópicos, antibióticos } \\
\text { sistêmicos, drenagem postural, repouso, higiene }\end{array}$ \\
\hline \multicolumn{6}{|l|}{ Manifestações crônicas } \\
\hline Infedema & muito baixa & $\begin{array}{l}\operatorname{sim}^{*} \\
\text { se infectado }\end{array}$ & NR & $\operatorname{sim}$ & $\begin{array}{l}\text { higiene diária, antibióticos e antifúngicos tópicos, } \\
\text { fisioterapia, drenagem postural, antibiótico sistêmico } \\
\text { profilático nos casos mais avançados }\end{array}$ \\
\hline linfo-escroto & variável & $\begin{array}{c}\operatorname{sim}^{*} \\
\text { se infectado }\end{array}$ & NR & $\operatorname{sim}$ & $\begin{array}{c}\text { higiene, antibióticos tópicos, sistêmicos (curativo ou } \\
\text { profilático), cirurgia reconstrutora }\end{array}$ \\
\hline hidrocele & variável & $\begin{array}{c}\operatorname{sim}^{*} \\
\text { se infectado }\end{array}$ & NR & $\operatorname{sim}$ & cirurgia \\
\hline quilocele & variável & $\begin{array}{c}\operatorname{sim}^{*} \\
\text { se infectado }\end{array}$ & NR & $\operatorname{sim}$ & cirurgia \\
\hline quilúria & variável & $\operatorname{sim}^{*}$ & & & \\
\hline & & se infectado & NR & $\operatorname{sim}$ & $\begin{array}{l}\text { dieta hipolipídica/hiperprotéica, hidratação, } \\
\text { repouso relativo, cateterização vesical }\end{array}$ \\
\hline adenopatia & baixa & $\begin{array}{c}\operatorname{sim}^{*} \\
\text { se infectado }\end{array}$ & NR & $\operatorname{sim}$ & higiene do membro ipsilateral \\
\hline EPT & $100 \%$ & $12-30$ dias & NR & $\operatorname{sim}$ & broncodilatadores, repouso \\
\hline
\end{tabular}

* $6 \mathrm{mg} / \mathrm{kg}$ em dose única

** LFA linfangite filarial aguda (morte de verme adulto)

*** DLAA dermatolinfangioadenite aguda (infecção bacteriana)

DEC = dietilcarbamazina; IV = ivermectina; NR = não recomendado

Tabela 2 - Esquemas terapêuticos em dose única recomendados a partir do $5^{\circ}$ ano de vida (quando a IV for administrada) para o tratamento em massa em regimes anuais ou semi-anuais.

\begin{tabular}{lcc}
\hline & \multicolumn{3}{c}{ Efeito terapêutico } \\
\cline { 2 - 3 } Droga/combinação (dose) & $\mathrm{VA}^{*}$ & $\mathrm{MF}$ \\
\hline $\mathrm{DEC} \mathrm{C}^{*}(6 \mathrm{mg} / \mathrm{kg})$ & $-/+$ & ++ \\
$\mathrm{IV}(400 \mu \mathrm{g} / \mathrm{kg})$ & - & +++ \\
$\mathrm{DEC}+\mathrm{IV}$ & $--/+$ & ++++ \\
$\mathrm{DEC}+\mathrm{ALB}(1$ comp. de 400mg) & $--/+$ & +++ \\
IV + ALB & - & ++++ \\
Sal fortificado com DEC & - & lento, mas eficaz \\
\hline
\end{tabular}

DEC: dietilcarbamazina; IV: ivermectina; ALB: albendazol; VA: verme adulto; MF: microfilária. * doses maiores não aumentam o efeito macrofilaricida. No caso do albendazol ainda é desconhecido. ** doses maiores aumentam o efeito microfilaricida. - sem efeito; -/+ efeito parcial; --/+ efeito parcial e reduzido pela combinação. ++;+++;++++: efeito crescente (as diferenças são pequenas entre as gradações, mas parecem ser significantes, principalmente no tratamento anual) 
Europa, na Austrália e nos Estados Unidos 68933444854 , embora nesses países o linfedema geralmente ocorra em conseqüência de malformação linfática, das complicações advindas de cirurgias e de radioterapia ${ }^{4} 43$.

O tratamento para linfedema de extremidades: a educação do paciente é uma etapa fundamental no tratamento do linfedema. Deve ser usada tanto para mudar os conceitos errôneos e fatalistas pré-existentes de que a progressão da doença é inevitável, quanto para ensinar os procedimentos corretos para os cuidados diários, encorajando e motivando o paciente a participar do programa de tratamento. Nas áreas endêmicas de filariose, onde as condições de higiene pessoal estão muito aquém das ideais, o mais importante é a prevenção dos episódios agudos bacterianos de repetição. Isso é feito através da higiene diária do membro afetado com água e sabão comum, objetivando a prevenção e a cura das portas de entrada, principalmente as interdigitais. Vale ressaltar a importância da higiene no membro contralateral, mesmo que este não esteja afetado. A grande maioria dos episódios agudos apresentados por esses pacientes se deve a surtos de infecções bacterianas recorrentes 212947525758 . Por isso, o tratamento tópico das lesões com cremes antibióticos e/ou antifúgicos pode ser necessário, principalmente em pacientes com doença mais avançada. São importantes também as medidas de melhoramento do retorno linfático e venoso que incluem a fisioterapia ativa e a drenagem postural (noturna e diurna). Adicionalmente, como recomendação individual, pode-se utilizar medidas compressivas. Durante os episódios bacterianos agudos (denominados de dermatolinfangioadenite aguda ou DLAA $)^{52}$, são de fundamental importância o uso de compressas frias no local afetado até o desaparecimento da dor, o repouso, a elevação do membro afetado e a antibioticoterapia sistêmica (oral ou parenteral, dependendo da severidade do quadro clínico), principalmente nas primeiras 48 horas após o início do quadro agudo. Os antiinflamatórios não devem fazer parte do tratamento pela sua ineficiência nesses casos. A cirurgia reconstrutora nos membros inferiores normalmente não é indicada, porém a intervenção para a retirada de nódulos ou formações verrucóides, principalmente os pediculados, ajuda não só a minimizar as potenciais portas de entrada, mas também a produzir um efeito cosmético em alguns pacientes.

O tratamento da linfangiomatose escrotal e do linfedema de genitália externa: o tratamento conservador da linfangiomatose escrotal ${ }^{26}$ (ou linfoescroto, como é conhecido em áreas endêmicas) do linfedema de saco escrotal e do pênis ${ }^{26}$ está, também, baseado na higiene e na cura das portas de entrada para infecções bacterianas, porém o seu grau de resposta é muito menor quando comparado ao resultado obtido para os membros inferiores e superiores. Assim, a cura só poderá ser obtida com a cirurgia reconstrutora (Tabela 1).
Material didático para uso individual está sendo produzido pela Organização Mundial da Saúde, em colaboração com o Center for Disease Control and Prevention (CDC) e a ONG Amaury Coutinho ${ }^{13}$, incluindo manuais e video de auto-ajuda para os pacientes.

O tratamento de outras formas crônicas: hidrocele, quilocele e quilúria: o tratamento da hidrocele e da quilocele é, eminentemente, cirúrgico. $\mathrm{O}$ tratamento de hidrocele através de instilação de substâncias esclerosantes na cavidade vaginal deve ser evitado, por não apresentar resultado uniformemente satisfatório ${ }^{12343840}$.

Na quilúria, o tratamento tem como ponto principal uma dieta hipolipídica, hiperprotéica e rica em líquidos. O repouso relativo está indicado nos casos mais graves ${ }^{64}$. O ideal seria que a dieta contivesse, principalmente, os triglicerídeos de cadeia média como fonte de lipídios ${ }^{37}$. A hidratação oral generosa é recomendada, com a finalidade de diminuir a ação irritativa da linfa no epitélio vesical e aumentar a diurese, diminuindo, assim, o risco de formação de trombos proteináceos que causam desconforto e dor miccional, inclusive podendo levar à retenção urinária aguda. Nesses casos, pode haver a necessidade do cateterismo vesical de alívio, de indicação mais comum nos pacientes do sexo masculino. Lembramos que, apesar da alta proteinúria, a qual chega até a 40 gramas nas 24 horas, o paciente não apresenta sintomatologia semelhante à síndrome nefrótica, pois a proteinúria é composta de fibronogênio/fibrina e não por albumina. O papel da cirurgia é controverso e de resultado imprevisível, sendo indicado somente como último recurso em casos severos que não respondem ao tratamento clínico ${ }^{41} 5961$. É importante, também, a monitorização da série vermelha e a correção nutricional da anemia que pode ocorrer pela perda crônica de sangue (microscópica, na maioria dos pacientes).

O tratamento comunitário. Através de medidas básicas de higiene, agora já se dispõe de uma forma de tratamento e prevenção em programas de saúde pública para os quase 15 milhões de pessoas que padecem de linfedema das extremidades, vivendo em áreas endêmicas de filariose. Pelo seu baixo custo, é de fácil aquisição para as populações carentes que constituem maioria nas regiões onde a filariose é endêmica. A experiência pioneira do Brasil, reproduzida no Haiti, no Egito, na Tanzânia, em Gana e na Índia, sugere que essa metodologia é factível para ser aplicada na comunidade através de um programa que foi chamado de Clube da Esperança ${ }^{14}$. Já estão disponíveis manuais e vídeos em diversos idiomas para serem distribuídos com os agentes de saúde nas diferentes áreas endêmicas em programas de campanhas de saúde pública.

Por outro lado, há um grande contingente de indivíduos vivendo em área endêmica que, apesar de já possuírem dano linfático subclínico e estarem ainda assintomáticos, podem beneficiar-se tremendamente do programa de prevenção do linfedema como parte de 
um programa de saúde pública. Isso seria possível através da educação das comunidades quanto à importância da higiene diária para aqueles indivíduos ainda sem doença aparente, principalmente os do sexo feminino. Idealmente, essa informação deve ser repassada para adolescentes como parte do currículo escolar ou através de outra forma eficaz de divulgação sistemática.

A estimativa é de que existam, pelo menos, cerca de 29 milhões de homens com manifestações clínicas urogenitais conseqüentes à doença bancroftiana, que incluem hidrocele, quilocele, linfedema de bolsa escrotal e pênis e linfoescroto. A hidrocele e a quilocele podem ser curadas através da cirurgia, procedimento este que depende da infra-estrutura de cada país em propiciar os meios para que esse tipo de atendimento seja feito à população em programas de saúde pública. Por outro lado, a complexidade para se dispor da infra-estrutura necessária para a realização da cirurgia reconstrutora, nos casos de elefantíase de pênis e escroto e linfoescroto, é muito maior do a que a necessária para o tratamento cirúrgico da hidro e da quilocele. Talvez a melhor, a mais prática e a menos onerosa opção para transpor essa dificuldade seja a criação de centros de referência regional de tratamento especializado. Esse modelo já funciona integralmente no Grande Recife, que tem o seu centro no Hospital das Clínicas da UFPE, através do Núcleo de Ensino, Pesquisa e Assistência em Filariose Linfática denominado de NEPAF ${ }^{60}$.

Sabe-se hoje que as crianças vivendo em área endêmica se infectam muito precocemente ${ }^{19} 45$, embora a grande maioria seja amicrofilarêmica. Por outro lado, dificilmente exibem doença clínica, exceto adenopatia ${ }^{25}$, apesar de a maior parte já apresentar doença subclínica evidenciada pela ultrassonografia ${ }^{23}$. Recomendamos que crianças e adolescentes apresentando manifestações clínicas, principalmente edema de membros e da genitália externa masculina, sejam avaliados por equipe multidisciplinar, para melhor definir a causa de sua doença, aventando-se entre os possíveis diagnósticos a possibilidade de malformação linfática congênita. Para o tratamento individual da infecção, preconizamos a dose única de $6 \mathrm{mg} / \mathrm{kg}$ de DEC. Nos programas de tratamento em massa, devemos incluir esse grupo etário, apesar de o mesmo contribuir bem menos para a transmissão da infecção. Contudo, esses indivíduos poderão, em muito, se beneficiar pela morte do verme adulto em uma idade mais precoce, o que diminui a possibilidade de progressão do dano linfático ${ }^{26}$. Em programas nos quais houver co-administração da DEC com ivermectina ou albendazol, as crianças em idade escolar serão os maiores beneficiários devido ao efeito anti-helmíntico adicional das duas últimas drogas ${ }^{53}$.

\section{REFERÊNCIAS BIBLIOGRÁFICAS}

1. Addiss DG, Beach MJ, Streit TG, Lutwick S, LeConte FH, Lafontant JG, Hightower AW, Lammie PJ. Randomised placebocontrolled comparison of ivermectin and albendazole alone and in combination for Wuchereria bancrofti microfilaremia in Haitian children. Lancet 350:480-484, 1997.

2. Albuquerque MFM, Marzochi MC, Ximenes R, Braga MC, Silva MCM, Furtado AF. Bancroftian filariasis in two urban areas of Recife, Brasil: The role of individual risk factors. Revista do Instituto de Medicina Tropical de São Paulo 37:225-233, 1995.

3. Andrade LD, Medeiros Z, Pires ML, Pimentel A, Rocha A, Figueredo-Silva J, Coutinho A, Dreyer G. Comparative efficacy of three different diethylcarbamazine regimens in lymphatic filariasis. Transactions of the Royal Society of Tropical Medicine and Hygiene 89:319-321, 1995.

4. Bertelli G, Venturini M, Forno G, Macchiavello F, Dini D. An analysis of prognostic factors in response to conservative treatment of postmastectomy lymphedema. Surgery, Gynecology and Obstetrics 175:455-460, 1992.

5. Bockarie MJ, Alexander ND, Hyun P, Dimber Z, Bockarie F, Ibam E, Alpers MP, Kazura JW. Randomised community-based trial of annual single-dose diethylcarbamazine with or without ivermectin against Wuchereria bancrofti infection in human beings and mosquitoes. Lancet 351:162-168, 1998.

6. Boris $\mathrm{M}$, Weindorf $\mathrm{S}$, Lasinski B, Boris $\mathrm{G}$. Lymphedema reduction by noninvasive complex lymphedema therapy. Oncology 8:95-106, 1994.

7. Brennan MJ. Lymphedema following the surgical treatment of breast cancer: a review of pathophysiology and treatment. Journal of Pain and Symptom Management 7:110-116, 1992.

8. Campisi C. A rational approach to the management of lymphedema. Lymphology 24:48-53, 1991.
9. Casley-Smith JR, Casley-Smith JR. Modern treatment of lymphoedema I. Complex physical therapy: The first 200 Australian limbs. Australian Journal of Dermatology 33:61-68, 1992.

10. Centers for Disease Control and Prevention. Recommendations of the International Task Force for Disease Erradication. Morbidity and Mortality Weekly Report 42 ( $\left.\mathrm{n}^{\circ} \mathrm{RR}-16\right): 1-38,1993$.

11. Coutinho A, Rocha A, Medeiros Z, Dreyer G. Eosinofilia Pulmonar Tropical Filariótica e o seu Diagnóstico Diferencial. Revista do Hospital das Clínicas da Faculdade de Medicina de São Paulo 53:42-51, 1998.

12. Dimberg M, Norlen H, Zachrisson L, Lindholm H. Testicular hydrocele treated by bismuth phosphate injection. International Urology and Nephrology 20:155-157, 1988.

13. Dreyer G. ONG Amaury Coutinho: transferência do conhecimento para o mundo real. Factível ou utopia? Revista da Sociedade Brasileira de Medicina Tropical 32:459-467, 1999.

14. Dreyer G, Addiss D. Hope Clubs: new strategy for lymphatic filariasis endemic areas. Transactions of the Royal Society of Tropical Medicine and Hygiene - Bulletin of Tropical Medicine and International Health 8:8, 2000.

15. Dreyer G, Addiss D, Norões J, Amaral F, Rocha A, Coutinho A. Ultrasonographic assessment of the adulticidal efficacy of repeated high-dose ivermectin in bancroftian filariasis. Tropical Medicine and International Health 1:427-432, 1996.

16. Dreyer G, Addiss D, Santos A, Figueredo-Silva J, Norões J. Direct assessment in vivo of the efficacy of combined single-dose ivermectin and diethylcarbamazine against adult Wuchereria bancrofti. Transactions of the Royal Society of Tropical Medicine and Hygiene 92:219-222, 1998. 
17. Dreyer G, Coutinho A, Miranda D, Norões J, Rizzo JA, Galdino E, Rocha A, Medeiros Z, Andrade LD, Santos A, Figueredo-Silva J, Ottesen EA. Treatment of bancroftian filariasis in Recife, Brazil: a two year comparative study of the efficacy of single treatments with ivermectin or diethylcarbamazine. Transactions of the Royal Society of Tropical Medicine and Hygiene 89:98-102, 1995.

18. Dreyer G, Dreyer P. Bases para o tratamento da morbidade em áreas endêmicas de filariose. Revista da Sociedade Brasileira de Medicina Tropical 33:217-221, 2000.

19. Dreyer G, Figueredo-Silva J, Carvalho K, Amaral F, Ottesen EA. Lymphatic filariasis in children: adenopathy and its evolution in two young girls. American Journal of Tropical Medicine and Hygiene 65: 204-207, 2001.

20. Dreyer G, Figueredo-Silva J, Neafie R, Addiss D. Lymphatic Filariasis. In: Nelson AM, Horsburg CR Jr (eds) Pathology of Emerging Infections 2. American Society for Microbiology, Washington DC, cap. 14, p. 317-342, 1998.

21. Dreyer G, Medeiros Z, Netto MJ, Leal NC, De Castro LG, Piessens WF. Acute attacks in the extremities of persons living in an area endemic for bancroftian filariasis: differentiation of two syndromes. Transactions of the Royal Society of Tropical Medicine and Hygiene 93:1-5, 1999.

22. Dreyer G, Norões J. Dietilcarbamazina no tratamento da filariose bancroftiana. Revista da Sociedade Brasileira de Medicina Tropical 30:229-240, 1997

23. Dreyer G, Noroes J, Addiss D, Santos A, Medeiros Z, FigueredoSilva J. Bancroftian Filariasis in a Paediatric Population: an Ultrasonographic Study. Transactions of the Royal Society of Tropical Medicine and Hygiene 93:633-636, 1999.

24. Dreyer G, Norões J, Amaral F, Nen A, Medeiros Z, Coutinho A, Addiss $D$. Direct assessment of the adulticidal efficacy of single dose ivermectin in bancroftian filariasis. Transactions of the Royal Society of Tropical Medicine and Hygiene 89:441-443, 1995.

25. Dreyer G, Noroes J, Figueredo-Silva J. Elimination of lymphatic filariasis as a public health problem. New insights into the natural history and pathology of bancroftian filariasis: implications for clinical management and filariasis control programs. Transactions of the Royal Society of Tropical Medicine and Hygiene 94:594596, 2000.

26. Dreyer G, Norões J, Figueredo-Silva J, Piessens WF. Pathogenesis of Lymphatic Disease in Bancroftian Filariasis: a Clinical Perspective. Parasitology Today 16:544-548, 2000.

27. Dreyer G, Norões J, Rocha A, Addiss D. Detection of living adult Wuchereria bancrofti in a patient with tropical pulmonary eosinophilia. Brazilian Journal of Medical and Biological Research 29:1005-1008, 1996

28. Dreyer G, Ottesen EA, Galdino E, Andrade L, Rocha A, Medeiros Z, Moura I, Cassimiro MI, Beliz MF, Coutinho A. Rena abnormalities in microfilaremic patients with bancroftian filariasis. American Journal of Tropical Medicine and Hygiene 46:745-751, 1992.

29. Dreyer G, Piessens W. Worms and microorganisms can cause lymphatic disease in residents of filariasis-endemic areas in lymphatic filariasis. In: Nutman TB (ed) Lymphatic Filariasis. $1^{\text {st }}$ edition, Imperial College Press, London, chap. 10, p. 245-264, 2000.

30. Dreyer G, Pires ML, Andrade LD, Lopes E, Medeiros Z, Tenorio J, Coutinho A, Norões J, Figueredo-Silva J. Tolerance of diethylcarbamazine by microfilaraemic and amicrofilaraemic individuals in an endemic area of bancroftian filariasis, Recife, Brazil. Transactions of the Royal Society of Tropical Medicine and Hygiene 88:232-236, 1994.

31. Dreyer G, Santos A, Norões J, Addiss D. Proposed panel of diagnostic criteria, including the use of ultrasound, to refine the concept of "endemic normals" in lymphatic filariasis. Tropical Medicine and International Health 4:575-579, 1999.

32. Figueredo-Silva J, Jungmann P, Norões J, Piessens WF, Coutinho C, Brito C, Rocha A, Dreyer G. Histological evidence for the adulticidal effect of low doses of diethycarbamazine in bancroftian filariasis. Transactions of the Royal Society of Tropical Medicine and Hygiene 90:192-194, 1996.

33. Földi E, Földi M, Clodius L. The lymphedema chaos: A lancet. Annals of Plastic Surgery 22:205-515, 1989.

34. Fuse H, Nishikawa Y, Shimazaki J, Katayama T. Aspiration and tetracycline sclerotherapy of hydrocele. Scandinavian Journal of Urology and Nephrology 25:5-7, 1991.

35. Gelband H. Diethylcarbamazine salt in the control of lymphatic filariasis. The American Journal of Tropical Medicine and Hygiene 50:655-662, 1994

36. Harb M, Faris R, Gad AM, Hafez ON, Ramzy R, Buck AA. The resurgence of lymphatic filariasis in the Nile delta. Bulletin of the World Health Organization 71:49-54, 1993.

37. Hashim SA. Treatment of chyluria and chylothorax with medium chain triglycerides. The New England Journal of Medicine 270:756-761, 1964.

38. Hu KN, Khan AS, Gonder M. Sclerotherapy with tetracycline solution for hydrocele. Urology 26:572-576, 1984.

39. Ismail MM, Jayakody RL, Weil GJ, Nirmalan N, Jayasinghe KSA, Abeyewickrema W, Sheriff MHR, Rajaratnam HN, Amaresekera N,Silva DCL, Michalski ML, Dissanaike AS. Efficacy of singledose combinations of albendazole, ivermectin, and diethylcarbamazine for the treatment of bancroftian filariais. Transactions of the Royal Society of Tropical Medicine and Hygiene 92:94-97, 1998.

40. Jimeno JMC, Garibay ASG, Ros FM. Hidrocele testicular: Terapeutica con esclerosantes. Archivos Espanholes de Urologia 42:109-110, 1989.

41. Kanetkar AV, Karanjawalla DK, Deshmukh SM, Sen PK. Initial experiences in the management of chyluria. Indian Journal of Surgery 28:381-389, 1966.

42. Kazura J, Greenberg J, Perry R, Weil G, Day K, Alpers M. Comparison of single-dose diethylcarbamazine and ivermectin for treatment of bancroftian filariasis in Papua New Guinea. The American Journal of Tropical Medicine and Hygiene 49:804-811, 1993.

43. Kissin MW, Querci della Rovere G, Easton D, Westbury G. Risk of lymphoedema following the treatment of breast cancer. The British Journal of Surgery 73:580-584, 1986.

44. Kobayashi MR, Miller TA. Lymphedema. Clinics in Plastic Surgery 14:303-313, 1987.

45. Lammie PJ, Reiss MD, Dimock KA, Streit TG, Roberts JM, Eberhard ML. Longitudinal analysis of the development of filarial infection and antifilarial immunity in a cohort of Haitian children. American Journal of Tropical Medicine and Hygiene 59:217-221, 1998.

46. Maizels RM, Denham DA. Diethylcarbamazine (DEC): immunopharmacological interactions of an anti-filarial drug. Parasitology 105:S49-S60, 1992. 
47. Montestruc E, Courmes E, Fontan R. Endemic lymphangitis in French Guiana and its relation to $W$. bancrofti filariasis. Indian Journal of Malariology 14:637-650, 1960.

48. Mortimer PS. Investigation and management of lymphoedema. Vascular Medicine Review 1:1-20, 1990.

49. Moulia-Pelat JP, Nguyen LN, Hascoet H, Luquiaud P, Nicolas L. Advantages of an annual single dose of ivermectin $400 \mu \mathrm{g} / \mathrm{kg}$ plus diethylcarbamazine for community treatment of bancroftian filariasis. Transactions of the Royal Society of Tropical Medicine and Hygiene 89:682-685, 1995.

50. Norões J. Aspectos clínicos, parasitológicos e histopatológicos decorrentes da morte de vermes adultos de Wuchereria bancrofti em linfáticos intra-escrotais. Tese de doutorado. Universidade Federal de São Paulo, São Paulo, SP, 2000.

51. Norões J, Dreyer G, Santos A, Mendes VG, Medeiros Z, Addiss $D$. Assessment of the efficacy of diethylcarbamazine on adult Wuchereria bancrofti in vivo. Transactions of the Royal Society of Tropical Medicine and Hygiene 91:78-81, 1997.

52. Olszewski WL, Jamal S, Manokaran G, Pani S, Kumaraswami V, Kubicka U, Lukomska B, Dworczynski A, Swoboda E, MeiselMikolajczyk F. Bacteriologic studies of skin, tissue fluid, lymph, and lymph nodes in patients with filarial lymphedema. The American Journal of Tropical Medicine and Hygiene 57:7-15, 1997.

53. Ottesen EA, Ismail MM, Horton J. The role of albendazole in programmes to eliminate lymphatic filariasis. Parasitology Today 15:382-386, 1999.

54. Pappas CJ, O'Donnell TF. Long-term results of compression treatment for lymphedema. Journal of Vascular Surgery 16:555-564, 1992.

55. Schofield FD, Rowley RE. The effect of prednisone on persistent microfilaremia during treatment with diethylcabamazine. American Journal of Tropical Medicine and Hygiene 10:849-854, 1961.
56. Seim AR, Dreyer G, Addiss D. Controlling morbidity and interrupting transmission: twin pillars of lymphatic filariasis elimination. Revista da Sociedade Brasileira de Medicina Tropical 32:325-328, 1999.

57. Shenoy RK, Kumaraswami V, Suma TK, Rajan K, Radhakuttyamma G. A double-blind, placebo-controlled study of the efficacy of oral penicillin, diethylcarbamaxine or local treatment of the affected limb in preventing acute adenolymphangitis in lymphoedema caused by brugian filariasis. Annals of Tropical Medicine and Parasitology 93:367-377, 1999.

58. Shenoy RK, Sandhya K, Suma TK, Kumaraswami V. A preliminary study of filariasis related acute adenolymphangitis with special reference to precipitating factors and treatment modalities. Southeast Asian Journal of Tropical Medicine and Public Health 26:301-305, 1995.

59. Singh M. Tropical parasitic infections of the urinary tract. In: Blandy J (ed) Urology. Blackwell, Oxford, p. 278-290, 1976.

60. Universidade Federal de Pernambuco. Boletin Oficial, Recife, 25 de outubro, 34:1-7, 1999.

61. Wickham JEA. Diseases of the renal artery, veins, and lymphatics. In: Blandy J (ed) Urology. Blackwell, Oxford, p. 369-374, 1976.

62. World Health Assembly. Fifitieth World Health Assembly: resolutions and decisions; annexes. World Health Organization (WHA50/1997/REC/1), Geneva, 5-14 May, 1997.

63. World Health Organization. Bridging the Gaps. World Health Report, Geneva, 1995.

64. Yu HYY. Chyluria. In: Husain I (ed) Tropical urology and renal disease. Churchill Livingstone, Edinburg, p. 296-304, 1984. 